\title{
PARASITISMO NATURAL DE OVOS DE TRIATOMÍNEOS POR TELENOMUS FARIAI LIMA, 1927 NO LABORATÓRIO
}

\author{
Alexandre José Fernandes, José Cândido da Silva e Liléia Diotaiuti
}

\begin{abstract}
Em novembro/89 verificou-se que o insetário do Centro de Estudos Emmanuel Dias Bambuí/MG apresentava alta infestação pelo microhimenóptero Telenomus fariai. Em 529 ovos de Panstrongylus megistus e Triatoma vitticeps examinados, $375(70,9 \%)$ apresentavam 1043 exemplares de $\mathrm{T}$. fariai, sendo $955(91,4 \%)$ fêmeas e $90(8,6 \%)$ machos. A seguir, verificou-se também o parasitismo de ovos de Triatoma infestans, e ausência de infestação de ovos de Rhodnius neglectus. As médias de parasitóide por ovo observadas foram de 7,9 em P. megistus; $8,7 \mathrm{em} \mathrm{T.}$ vitticeps $e 10 \mathrm{em}$ T. infestans. A importância do relato deve-se à possibilidade da infestação de colônias mantidas para fins de pesquisa a partir da introducão em insetários de ovos procedentes do campo, com o estabelecimento de altas taxas de infestação e acentuado declínio da criação. $O$ isolamento e a eliminação dos parasitóides pelo manejo dos ovos parasitados e vedação dos frascos do insetário com tecido de malha estreita (em torno de $0,25 \mathrm{~mm}$ ) mostraram-se eficientes no controle do $\mathrm{T}$. fariai.
\end{abstract}

Palavras-chaves: Telenomus fariai. Parasitóide. Triatomíneo.

O Telenomus fariai é um microhimenóptero da familia Scelionidae, subfamília Telenominae, descrito por Lima 4 parasitando ovos de Panstrongylus megistus procedentes de Lassance, Minas Gerais. Estudos de sua biologia ${ }^{5}$ mostraram que o período médio de vida desses insetos é de 30 dias, sendo que as fêmeas são as que vivem mais tempo. Estas, fecundadas ou não, iniciam a oviposição em ovos de triatomíneos horas depois de terem nascido. Com uma temperatura média de $19-20^{\circ} \mathrm{C}$ verificou-se que, da postura à saída das formas adultas, decorrem de 27 a 35 dias. Segundo Lima $^{5}$, as fêmeas não fecundadas geram por partenogênese apenas insetos do sexo masculino. Os machos que são gerados por fêmeas fecundadas medem de 0,78 a $0,80 \mathrm{~mm}$, enquanto os partenogenéticos medem, semelhante às fêmeas, de 1,08 a $1,18 \mathrm{~mm}$ de comprimento.

Outros autores confirmaram, posteriormente, o achado de $\mathrm{Lima}^{4}$, encontrando ovos naturalmente

Centro de Pesquisas René Rachou/FIOCRUZ, Belo Horizonte, MG. Centro de Estudos "Emmanuel Dias", Bambui, MG.

Departamento de Parasitologia, Instituto de Ciências Biológicas/ Universidade Federal de Minas Gerais, Belo Horizonte, MG.

Endereço para correspondência: Dr. Alexandre José Fernandes. Centro de Pesquisas René Rachou. CP: 1743 - 30190 BeloHorizonte, MG, Brasil.

Recebido para publicaçāo em 04/06/90. parasitados de Triatoma infestans 6 na Argentina, de Triatoma pallidipenis ${ }^{7}$ no México, de Triatoma dimidiata $^{10}$ na Costa Rica. Em Minas Gerais, Brasil, Pellegrino ${ }^{8}$ encontrou o parasitismo natural de ovos de $T$. infestans e $P$. megistus procedentes de várias localidades, entre elas o município de Bambui.

\section{MATERIAL E MÉTODOS}

Em novembro de 1989 verificou-se que o insetário do Centro de Estudos "Emmanuel Dias" Fundação Oswaldo Cruz, apresentava uma alta infestação pelo microhimenóptero T. fariai, com elevado número de ovos de $P$. megistus e Triatoma vitticeps parasitados por diferentes formas evolutivas daquele inseto. Todos os ovos destes triatomíneos foram coletados e acondicionados em frascos de vidro bem vedados, para que fosse observado o número de ovos parasitados, e de fêmeas e machos do microhimenóptero. Pesquisou-se ainda a possibilidade de parasitismo dos demais triatomíneos existentes no insetário (Rhodnius neglectus e T. infestans). Estes ovos foram acompanhados diariamente por 40 dias, passando a ser acondicionados individualmente em tubos de hemólise aqueles que apresentavam indício de parasitismo, para verificação do número de microhimenópteros e proporção de machos e fêmeas destes insetos por ovo.

Em seguida, como medidas de controle, os frascos contendo triatomineos adultos foram vedados 
com tecido de malha estreita (em torno de $0,25 \mathrm{~mm}$ ) e todos os ovos passaram a ser coletados e mantidos em quarentena para eliminação daqueles que estivessem parasitados. Dois meses após, procedeu-se uma avaliação deste procedimento pela observação da presença ou não do parasitismo em 430 ovos de $P$. megistus, 230 de $T$. infestans e 110 de $T$. vitticeps.

\section{RESULTADOS}

Aproximadamente 40 dias após os ovos dos triatomíneos terem sido coletados e acondicionados em frascos de vidro, todos os microhimenópteros atingiram o estádio adulto, quando foi observado o número de ovos parasitados, e de fêmeas e machos. De 529 ovos, $375(70,9 \%)$ estavam parasitados, dando origem a 1045 exemplares de $T$. fariai, sendo 955 $(91,4 \%)$ fêmeas e $90(8,6 \%)$ machos.

Na Tabela 1, pode-se observar o número de microhimenópteros e proporção entre machos e fêmeas deste inseto em ovos de $P$. megistus, $T$. vitticeps e $T$. infestans. Não foi observado parasitismo de ovos de $\boldsymbol{R}$. neglectus.

Em 770 ovos de triatomineos (P. megistus, $T$. infestans e $T$. vitticeps) coletados dois meses após terem sido iniciadas as medidas de controle, não observou-se nenhum parasitado pelo microhimenóptero.

\section{DISCUSSÃO}

A importância do parasitismo de ovos de triatomíneos pelo T. fariai aqui assinalado deve-se à possibilidade de estabelecimento de altas taxas de infestação (como a de $70,9 \%$ observada antes da instalação das medidas de controle), em função do elevado número de microhimenópteros produzidos por ovo $(8,1$ parasitóide/ovo), e pelo curto periodo neces- sário para que se complete o ciclo evolutivo destes insetos (média de 38 dias, segundo Pellegrino ${ }^{9}$ ).

Os estudos do parasitismo experimental pelo $T$. fariai de ovos de várias espécies de triatomíneos realizados por Pellegrino ${ }^{9}$ e Zeledón ${ }^{11}$ mostraram, de forma semelhante ao observado em Bambuí neste trabalho, que o número de microhimenópteros que emergem de ovos parasitados varia, dependendo da espécie de triatomineo. Este fato, como assinala Lima $^{5}$, depende do tamanho do ovo parasitado. Com relação à proporção entre os sexos de $T$. fariai, pôdese observar, como já assinalado na literatura ${ }^{9}$, que o número de fèmeas por ovo parasitado é maior que ao dos machos. A percentagem média dos sexos do microhimenóptero foi de $91,4 \%$ de fêmeas e $8,6 \%$ machos, ou seja, aproximadamente 1 macho para 7 fềmeas.

O parasitismo de ovos de triatomíneos pelos microhimenópteros Telenomus costalimai (Scelionidae) e Ooencyrtus trinidadensis (Encyrtidae) foi experimentalmente estudado por Feleciangeli 3 na Venezuela. A autora verificou especificidade do $T$. costalimai ao gènero Rhodnius, frente aos demais estudados (Triatoma, Eratyrus e Panstrongylus). Ovos de várias espécies de triatomineos, incluindo de $R$ neglectus, $R$. prolixus, $R$. pallescens e $R$. neivai, foram experimentalmente expostos ao T. fariai ${ }^{11}$, não sendo observado nenhum parasitóide nos ovos das espécies de Rhodnius. A ausência de parasitismo de $R$ neglectus (Tabela 1 ) poderia relacionar-se a fenômeno de especificidade parasitária, à semelhança do descrito por Feliciangeli ${ }^{3}$ e Zeledón ${ }^{11}$. Na possibilidade de parasitismo de $R$. neglectus pelo $T$. fariai, a ausência de parasitismo dos ovos desta espécie no insetário de Bambui poderia explicar-se pelo fato das tampas dos seus frascos serem envoltas em tecido de malha estreita, por medida de segurança, uma vez que os ovos deste triatomíneo são também colocados aderidos ao tecido. Desta forma, não haveria contato

Tabela 1 - Parasitismo natural de ovos de triatomíneo pelo Telenomus fariai no insetário do Centro de Estudos "Emmanuel Dias"-FIOCRUZ, Bambuí, Minas Gerais.

\begin{tabular}{lccccc}
\hline & $\begin{array}{c}\text { No } \\
\text { ovos } \\
\text { obtidos }\end{array}$ & $\begin{array}{c}\text { No ovos } \\
\text { parasitados } \\
(\%)\end{array}$ & $\begin{array}{c}\text { Média } \\
\text { parasitóide } \\
\text { /ovo }\end{array}$ & $\begin{array}{c}\text { Média } \\
\text { adultos/ovo }\end{array}$ \\
\hline P. megistus & 448 & $26(5,8)$ & 7,9 & 0,9 & 7,0 \\
$T$. vitticeps & 315 & $7(2,2)$ & 8,7 & 0,9 & 7,8 \\
$T$. infestans & 100 & $1(1,0)$ & 10,0 & 1,0 & 9,0 \\
$R$. neglectus & 100 & 0 & 0 & 0 & 0 \\
\hline Total & 963 & $34(3,5)$ & 8,1 & 0,9 & 7,3 \\
\hline
\end{tabular}


Fernandes AJ, Silva JC, Diotaiuti L. Parasitismo natural de ovos de triatomineos por Telenomus fariai Lima, 1927 no laboratório. Revista da Sociedade Brasileira de Medicina Tropical 23: 149-151, jul-set, 1990.

das fêmeas de Telenomus com os ovos destes triatomineos.

A origem do parasitismo aqui descrito, provavelmente, se deveu à introdução no insetário de ovos de triatomíneos parasitados, trazidos pela população ao Centro de Estudos "Emmanuel Dias" através do Programa de Vigilância Epidemiológical, uma vez que já foi constatado o parasitismo natural de ovos de P. megistus e T. infestans no município de Bambui 8 .

As medidas de controle, nestas circunstâncias, restritas à eliminação dos ovos parasitados e vedação dos frascos contendo os adultos de triatomineos, mostraram-se eficazes, uma vez que dois meses após implantadas não mais observou-se a presença do $T$. fariai no insetário.

\section{SUMMARY}

In november/89 in the insectary of Centro de Estudos "Emmanuel Dias"/Bambuí-MG a high infestation by the microhymenoptera T. fariai was found. A mong the 529 eggs examined from $\mathrm{P}$. megistus and $\mathrm{T}$. vitticeps, 375 (70.9\%) of them showed a total of 1045 parasites $(91.4 \%$ females and $8.6 \%$ males). Later on it was found that $\mathrm{T}$. infestans eggs were also parasitised by $\mathbf{T}$. fariai but not those from $\mathbf{R}$. neglectus. The observed parasitoid/egg average was 7.9 in $\mathrm{P}$. megistus; 8.7 in $\mathrm{T}$. vitticeps and 10 in $\mathrm{T}$. infestans. The present report has special importance considering the real possibility of $\mathrm{T}$. fariai infestation due to the access of infested triatomine eggs from field captures inducing great damage to colonies maintained for research purposes. These eggs have to be carefully examined, isolated and eliminated. This procedure and the closing of triatomine containers with thin net $(0.25 \mathrm{~mm})$ is show to be efficient in $\mathbf{T}$. fariai control.

Key-words: Telenomus fariai. Parasitoid. Triatomine.

\section{REFERÊNCIAS BIBLIOGRÁFICAS}

1. Dias JCP, Garcia ALR. Vigilancia epidemiologica con participación comunitaria. Un programa de enfermedad de Chagas. Boletín de la Oficina Sanitaria Panamericana 84:533-544, 1978.

2. Feliciangeli MD. Experimental parasitism of triatomine eggs (Hemiptera: Reduviidae) by the microhymenoptera Ooencyrtus trinidadensis venatorius (Chalcidoidea: Encyrtidae) and Telenomus costalimai (Proctotrupoidea: Scelionidae). Journal of Medical Entomology 15: 25-30, 1978.

3. Feliciangeli de Pin̂ero D. Comportamiento del microhimenóptero Telenomus costalimai. Boletin de la Dirección de Malariologia y Saneamento Ambiental 18: 38$42,1978$.

4. Lima AC. Nota sobre o Telenomus fariai, novo scelionideo, parasito endophago dos ovos de Triatoma megista (Burm.). Sciencia Medica 5: 450-542, 1927.

5. Lima AC. Notas sobre a biologia do Telenomus fariai Lima, parasito dos ovos de Triatoma. Memórias do Instituto Oswaldo Cruz 21: 201-209, 1928.

6. Mazza S. Jörg ME. Tercera nota sobre Triatomidae (Hemiptera Heteroptera Reduvioidae). Investigaciónes sobre la enfermedad de Chagas 36: 26-58, 1938.

7. Peláez D. Algumas notas sobre el hallazgo en México de un microhimenóptero parásito de huevos de Triatoma pallidipennis (Stal). Sciencia 5: 29-33, 1944.

8. Pellegrino J. Nota sobre o parasitismo de ovos de Triatoma infestans e Panstrongylus megistus pelo microhimenóptero Telenomus fariai Lima, 1927. Memórias do Instituto Oswaldo Cruz 48: 669-673, 1950.

9. Pellegrino J. Parasitismo experimental de ovos de várias espécies de Triatoma pelo microhimenóptero Telenomus fariai Lima, 1927. Memórias do Instituto Oswaldo Cruz 48: 675-686, 1950.

10. Zeledón $\mathrm{R}$. Sobre la biología del Telenomus fariai Lima, 1927 (Hymenoptera: Scelionidae), parásito endófago de huevos de algunos Triatominae. Revista de Biologia Tropical 5: 1-17, 1957.

11. Zeledón R. Enemigos naturales. In: El Triatoma dimidiata (Latreille, 1811) y su relación con la enfermedad de Chagas, San José, Costa Rica, p. 98-127, 1981. 